\title{
Quantifying Phase Behaviour in Model Food Composites Using 3D Confocal Laser Scanning Microscopy
}

\author{
Pranita Mhaske $^{1} \cdot$ Stefan Kasapis $^{1} \cdot$ Asgar Farahnaky $^{1} \cdot$ Mina Dokouhaki $^{1}$ (])
}

Received: 27 September 2021 / Accepted: 27 December 2022 / Published online: 10 January 2022

(c) Crown 2022

\begin{abstract}
There is an increasing demand for the design of complex bio-composites with customized structural characteristics for use in processed food products. Phase behaviour of these mixtures determines textural properties, encouraging the pursue of a rapid technique that can accurately quantify it. The present work tests the efficacy of confocal laser scanning microscopy (CLSM) coupled with image analysis software (Imaris), for the quantification of phase behaviour in complex tertiary systems. In doing so, it develops phase separated gels of agarose and gelatin supporting inclusions of canola oil. The polysaccharide was replaced with whey protein isolate (WPI) and the topology of the tertiary dispersion with gelatin and canola oil was also examined. Reproducible phase volume estimates were obtained, including those of the lipid phase, which were a close match to the actual concentrations added to the hydrocolloid gel. The approach could offer an alternative to the rheological estimation, via theoretical blending law analysis, of phase volumes in bio-composites.
\end{abstract}

Keywords 3D imaging $\cdot$ Image analysis $\cdot$ Phase behaviour $\cdot$ Confocal laser scanning microscopy

\section{Introduction}

Complex biomaterial mixtures are used increasingly in added value food, nutraceutical and pharmaceutical industries to design materials of enhanced techno-functionality. A thorough understanding of the structure-function relationship of components in these composites is crucial for the successful fabrication of materials with multifaceted properties to suit a variety of industrial applications [1].

Of the many techniques used for studying structure-function dynamics, rheology-based blending law analysis remains one of the most popular. Borrowed from the realm of synthetic polymer science, blending laws probe phase behaviour and characterize water partition in composites made of two gelling biopolymers. This is achieved by following the mechanical properties of the constituent polymeric phases in relation to the overall structure of the binary mixture, as outlined first by Takayanagi et al. and Nielsen [2, 3]. Though the theoretical blending laws are robust, they are

Mina Dokouhaki

mina.dokouhaki@rmit.edu.au

1 School of Science, RMIT University, Bundoora West Campus, Plenty Road, Melbourne, VIC 3083, Australia an indirect method of estimating phase volume and require considerable experimentation and modelling expertise.

Confocal laser scanning microscopy (CLSM) is widely used in biosciences and biomedical sciences for qualitative analysis of fluorescent nanomaterials, orthopedic implant applications, etc. It might be a promising technique for direct quantification of phase volumes in biomaterial composites when paired with rapidly advancing image processing. CLSM allows sample analysis in its native state without a pre-processing step that other microscopic techniques need like fixation, sectioning and/or freeze-drying. Multiple twodimensional (2D) images can be captured along the Z-axis by moving the focal plane in steps of specified thickness through the sample depth. This Z-stack feature of the confocal microscopy offers a unique insight into a gel's true 3D microstructure [4].

Recently, a novel technique combining CLSM and image analysis was developed to quantify phase volumes in model binary systems comprising agarose and canola oil, and subsequently in more complex bio-composites of agarose-ghee and agarose-microcrystalline cellulose [5-7]. The combined CSLM/image analysis protocol dealt with the scaffolding complexity in these biomaterials, with phase volume estimates matching well the predictions of the rheology-based blending law analysis. 
Such successes of the microscopic protocol, in combination with the continuous advancement in imaging and image analysis techniques, is encouraging for application in a plethora of fields. It could be used to observe structural changes at the nanoscale for tracking and quantifying drug delivery, solid-state diffusion, etc. [8]. With the inclusion of an additional dimension, time, it might be possible to visualise and quantify dynamic changes in the phase behaviour of composites during gelation upon storage [9]. Exploiting the capability of CLSM to excite multiple fluorophores simultaneously, might offer an avenue of accurately differentiating between multiple constituents of a composite system provided they are adequately labelled [10].

The aforementioned discussion suggests that the combined microscopic/image analysis protocol should be used to capture and quantify images of further complexity, e.g. from tertiary or even higher order composites. The present work, therefore, develops a microscopy-based experimental methodology that deals with the phase behaviour in tertiary bio-composites, as a rapid analytical technique.

\section{Materials and Methods}

\section{Materials}

Type- 1 agarose comprising $10 \%$ moisture, $0.25 \%$ ash and $0.15 \%$ sulphate was purchased from Sigma-Aldrich (Sydney, Australia). Type-B bovine gelatin with a bloom value of 225 and a molecular weight of about $173 \mathrm{kDa}$ was also supplied by Sigma-Aldrich. Whey protein isolate was supplied by Fonterra (Auckland, New Zealand). The powder had $94.12 \%$ protein (dry basis), $4.64 \%$ moisture, $0.18 \%$ fat and $0 \%$ lactose content according to the supplier specifications. Canola oil, purchased from Manildra (Gladesville, Australia), had a density of $0.918 \mathrm{~g} / \mathrm{ml}$.

The stains used for confocal microscopy were all procured from Sigma-Aldrich (Sydney, Australia). 5-DTAF (5-(4, 6-Dichlorotriazinyl) Aminofluorescein), Fluorescein isothiocyanate (FITC), and Rhodamine-B were used to stain agarose, gelatin and canola oil, respectively. Reagents used for staining, i.e. $99 \%$ pure crystalline $\mathrm{Na}_{2} \mathrm{SO}_{4}, 98 \%$ anhydrous $\mathrm{NaOH}$ pellets, $99.9 \%$ dimethyl sulfoxide (DMSO) and pure ethanol were also purchased from Sigma-Aldrich.

\section{Methods}

Staining Before preparing the samples, agarose was covalently labelled with 5-DTAF using the method by Mhaske et al. [7]. Briefly, $1 \mathrm{~g}$ of agarose was added to $50 \mathrm{ml}$ milliQ water and heated to $80{ }^{\circ} \mathrm{C}$ with constant stirring. After the complete solvation of agarose, the solution was cooled down to $60{ }^{\circ} \mathrm{C}$ before adding $8 \mathrm{mg}$ of 5 -DTAF. Then, $15 \mathrm{ml}$ of
$1 \%$ (w/v) $\mathrm{Na}_{2} \mathrm{SO}_{4}$ was slowly added, followed by 3-4 drops of $10 \%(\mathrm{w} / \mathrm{v}) \mathrm{NaOH}$. Following continuous stirring for $2 \mathrm{~h}$, $120 \mathrm{ml}$ of absolute ethanol was added to observe a fluorescent yellow precipitate. The dispersion was kept at $-20{ }^{\circ} \mathrm{C}$ for 20 min before filtering it and washing the residue with absolute ethanol. The residue was kept in a desiccator till it was completely dry and then ground using a mortar and pestle to obtain a fine yellow powder of covalently labelled agarose. Canola oil was stained with a lipophilic stain, Rhodamine-B. Stock solution of Rhodamine-B $(0.01 \mathrm{~g} / \mathrm{ml})$ was prepared using DMSO and the required amount was added to canola oil to obtain a final concentration of $0.005 \mathrm{~g} / \mathrm{L}$.

Sample Preparation Two tertiary systems were prepared, as follows: sample 1 , containing $3 \%(\mathrm{w} / \mathrm{w})$ agarose plus $2 \%(\mathrm{w} / \mathrm{w})$ gelatin and $2 \%(\mathrm{w} / \mathrm{w})$ canola oil, and sample 2 comprising $10 \%(\mathrm{w} / \mathrm{w})$ gelatin plus $5 \%(\mathrm{w} / \mathrm{w})$ whey protein isolate (WPI) and 5\% (w/w) canola oil. To prepare sample $1,3 \mathrm{~g}$ of covalently labelled agarose was added to $93 \mathrm{ml}$ of milliQ water, heated to $70^{\circ} \mathrm{C}$ and stirred until complete solvation. The solution was cooled to $60{ }^{\circ} \mathrm{C}$ before adding $2 \mathrm{~g}$ of gelatin. The binary mixture was further cooled to $50{ }^{\circ} \mathrm{C}$ before adding $2 \mathrm{~g}$ of Rhodamine-B stained canola oil. The mixture was agitated vigorously to prevent coalescence of the oil droplets. About $1 \mathrm{ml}$ of sample was carefully pipetted out and transferred into the well of Coverwell Press-Seal imaging chamber that was precooled to $5{ }^{\circ} \mathrm{C}$ to ensure rapid and homogenous in-situ gelation. The imaging chamber well was $2.8 \mathrm{~mm}$ deep and had a $20 \mathrm{~mm}$ diameter (SigmaAldrich, Sydney, Australia). For sample 2, $10 \mathrm{~g}$ gelatin was added to $80 \mathrm{ml}$ of milliQ water and heated to $60^{\circ} \mathrm{C}$ with constant stirring. The solution was cooled to $40{ }^{\circ} \mathrm{C}$ before adding $0.001 \%$ FITC. It was stirred for 60 min to stain the protein. $5 \mathrm{~g}$ WPI was slowly added into the solution and stirred till it was homogenously dispersed. Finally, $5 \mathrm{~g}$ of Rhodamine-B stained canola oil was added to the mixture and stirred vigorously. $1 \mathrm{ml}$ sample was transferred into the precooled imaging chamber well and allowed to gel rapidly.

Image Acquisition and Quantification An inverted ECLIPSE Ti-E microscope (Tokyo, Japan) fitted with a Nikon A1R laser was employed to capture confocal images at 10x. A $488 \mathrm{~nm}$ laser was used to excite the 5-DTAF labelled agarose in sample 1 and the FITC-labelled gelatin in sample 2. Emissions were captured between 500 and $530 \mathrm{~nm}$. The Rhodamine-B stained canola oil was excited at $561 \mathrm{~nm}$ and emissions were captured between 570 and $620 \mathrm{~nm}$. Each CLSM image was captured is an 8-bit image of $1279 \mu \mathrm{m} \times 1279 \mu \mathrm{m}$ in size. Twenty-four images were obtained along the Z-axis at an interval of $12 \mu \mathrm{m}$ for both samples. These images were analysed with Bitplane's image analysis software, Imaris 9.1.0 (Belfast, North Ireland) using the method previously described by Mhaske et al. [5]. The 'Surfaces' feature was 
Fig. 1 (a) 2D, (b) Z-stack, and (c) 3D CLSM images of 3\% (w/w) agarose plus $2 \%(w / w)$ gelatin and $2 \%(\mathrm{w} / \mathrm{w})$ canola oil. The agarose, gelatin and oil phases are denoted in green, black and red, respectively. The composite 3D CLSM image is further split to denote $3 \mathrm{D}$ images of the constituents (d) agarose and gelatin, and (e) oil phase separately (Images captured at 10x, scale bar denotes $200 \mu \mathrm{m})$
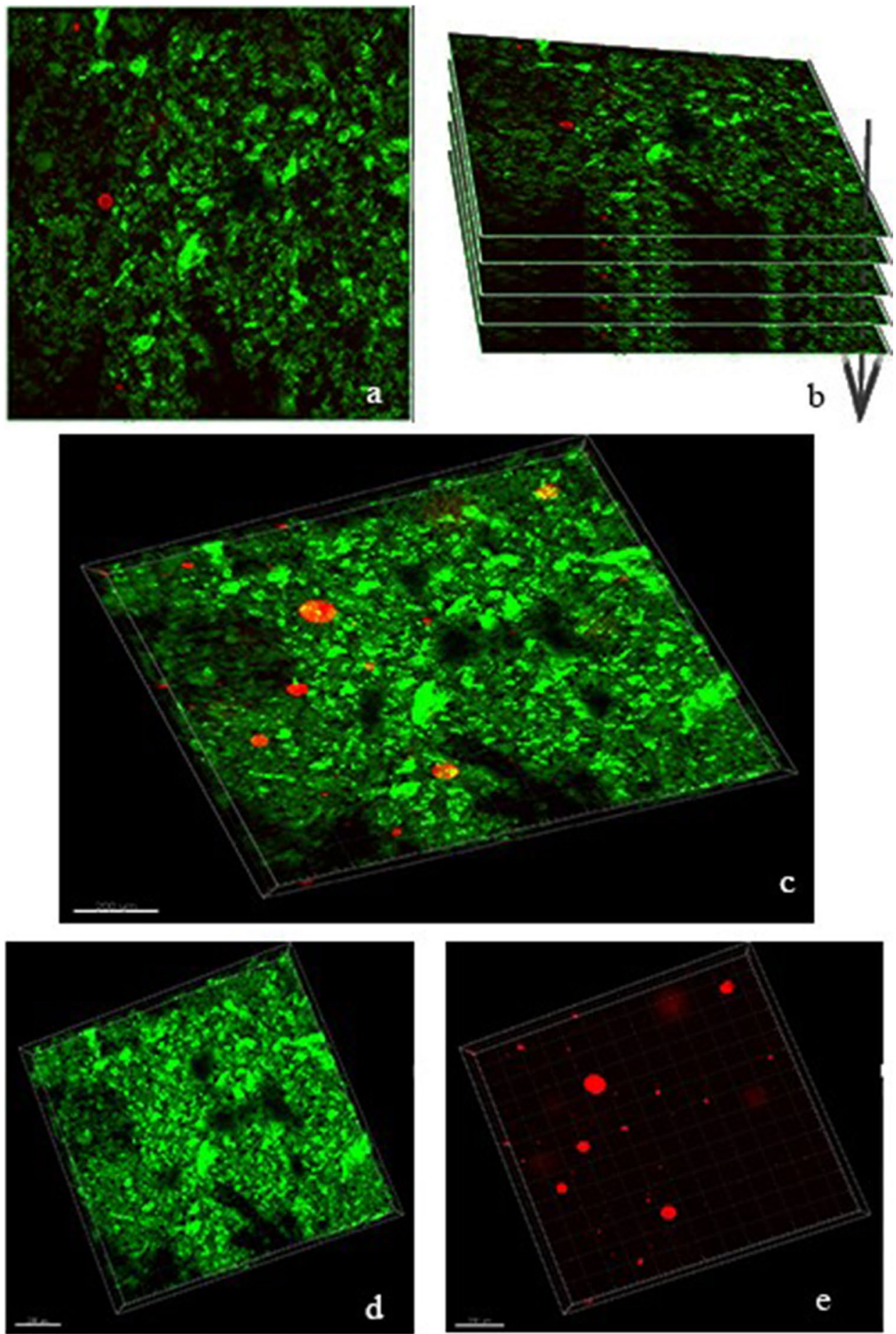

chosen to open the Z-stack and the background intensity was subtracted to reduce noise and smoothen the image. The generated 3D image was quantified volumetrically to obtain the volume of each phase in both samples. A Z-stack of 2D images from three different sections across both samples was captured to compensate for the effect of any inhomogeneous distribution of the individual phases, if any.

\section{Statistical Analysis}

Volume measurements of the different phases were carried out in triplicate and data is expressed as mean \pm standard deviation. Results were evaluated by one-way analysis of variance (ANOVA) using Minitab 18 (Minitab Inc., Pennsylvania, USA). Significance differences were defined as $\mathrm{p}<0.05$ with the Tukey test. 


\section{Results and Discussion}

In order to test the efficacy of the combined microscopic/ image analysis protocol in quantifying the phase behaviour of tertiary dispersions, we opted for two hydrocolloid/lipid systems of distinct constituents and concentrations. In terms of structural functionality, the three components of the two composites were selected to include immiscible, hygroscopic and gel-forming properties. Another consideration of selection was that the components of the mixture could be individually stained with dyes that exhibited no overlapping between their excitation and emission wavelengths.

Figure 1a represents a two-dimensional (2D) image of Sample 1, containing 3\% (w/w) agarose polysaccharide
Fig. 2 (a) 2D, (b) Z-stack, and (c) 3D CLSM images of $10 \%$ $(\mathrm{w} / \mathrm{w})$ gelatin plus $5 \%(\mathrm{w} / \mathrm{w})$ WPI and 5\% (w/w) canola oil. The gelatin, WPI and oil phases are denoted in red, black and green, respectively. The composite 3D CLSM image is further split to denote 3D images of the constituents (d) gelatin and WPI, and (e) oil phase separately (Images captured at 10x, scale bar denotes $200 \mu \mathrm{m}$ )
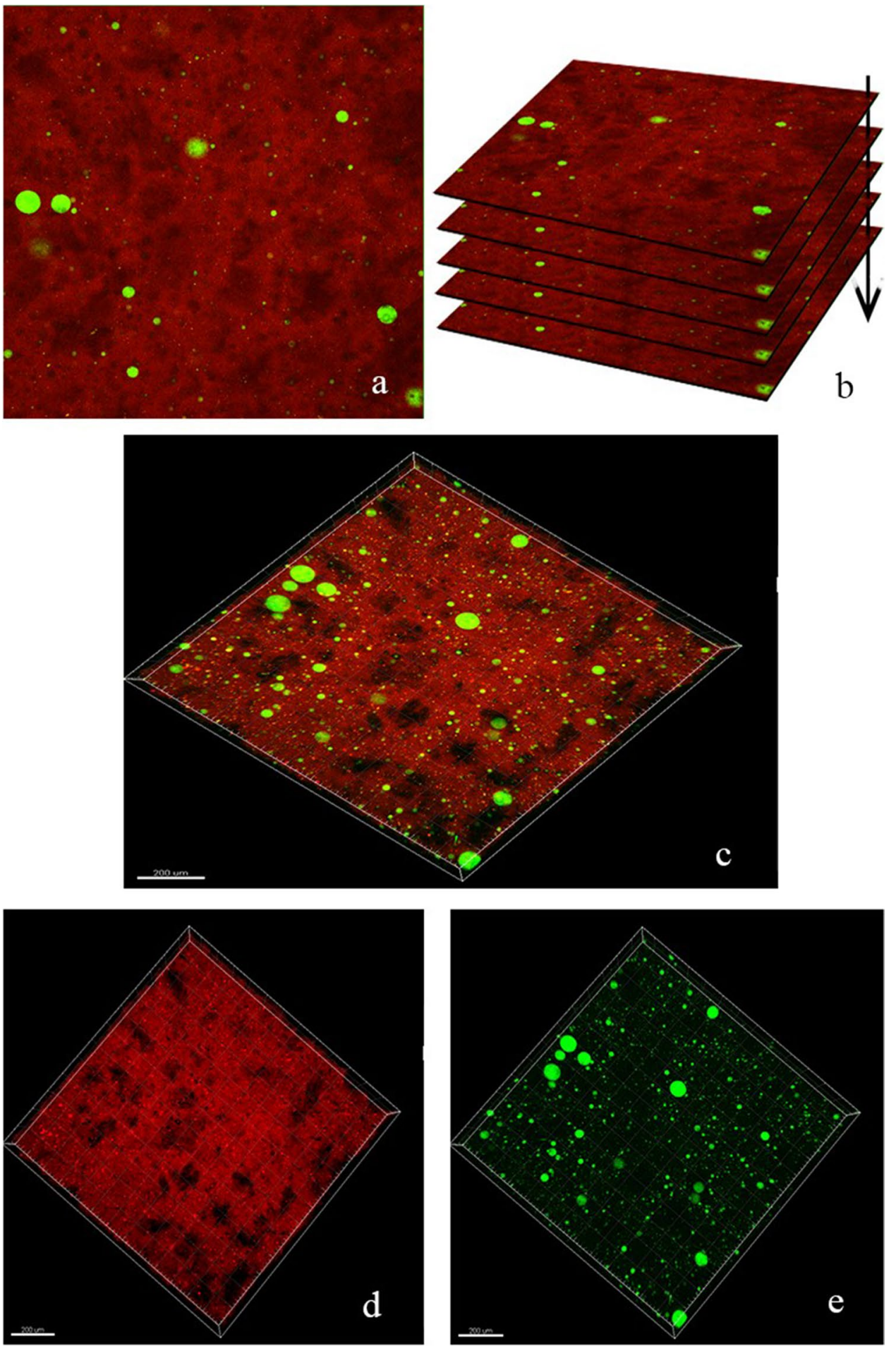
plus $2 \%(\mathrm{w} / \mathrm{w})$ gelatin protein and $2 \%(\mathrm{w} / \mathrm{w})$ canola oil (lipid phase). Clear microscopic domains are obtained for the polymeric and lipid components of our mixture. The stained agarose and lipid phases appear to be green and red, respectively, and the unstained gelatin is denoted in black. It was observed that the agarose is the continuous phase within which the gelatin and lipid phases form discontinuous inclusions.

Figure $2 \mathrm{a}$ depicts the two-dimensional image of another tertiary composite, i.e. Sample 2. This is made of $10 \%$ (w/w) gelatin plus $5 \%(\mathrm{w} / \mathrm{w})$ whey protein isolate (WPI) and $5 \%(\mathrm{w} / \mathrm{w})$ canola oil. The stained gelatin and oil phases show in the 2D image as red and green, respectively, whereas the WPI phase appears to be black. In this case, the discontinuous WPI and canola oil filler particles are dispersed within in the continuous gelatin matrix. For both Samples 1 and 2, multiple 2D images were captured along the Z-axis of the gelled matrix to obtain a dense Z-stack shown in Figs. $1 \mathrm{~b}$ and $2 \mathrm{~b}$, respectively. These Z-stacks are then utilised to generate $3 \mathrm{D}$ images by employing the image processing software, Imaris, as shown in Fig. 1c for Sample 1 and Fig. 2c for Sample 2.

Image analysis in Imaris (and other contemporary image analysis software) allows the user to split images based on the wavelength used for illumination, making it possible to view and generate new images of the distribution of specific components in the composite matrix. We were able then to split Fig. 1c into Figs. 1d and e, which are the three-dimensional (3D) images of the polymeric (agarose and gelatin) and lipid (canola oil) phases, respectively. In a similar vein, Fig. 2c was split with Imaris into Figs. 2d and e, which denote the 3D images of the distribution of polymeric (gelatin and WPI) and the lipid (canola oil) phases, respectively, within the composite gel.

Subsequent analysis allowed quantification of these images with the microscopic protocol that we developed for Imaris using the marching cube algorithm, as described in the experimental section. The algorithm identifies the object voxels, creates a polygonal mesh around it to encompass the identified boundary and then estimates the volume of the object entrapped in the mesh [11]. Thus, phase volume estimates obtained by processing Figs. 1d, e, $2 \mathrm{~d}$ and e for the two tertiary composites are summarised in Table 1. The phase volume of canola oil in the mixture depends entirely on the added concentrations (2\%, w/w, in Sample 1 and 5\%, w/w, in Sample 2), as there is no water molecule partitioning between the aqueous and lipid phases. Gratifyingly, Table 1 demonstrates a close agreement in the oil phase volume estimates generated by Imaris $(1.9 \%$, w/w, in Sample 1 and $5.1 \%$, w/w, in Sample 2 ) and the actual concentration of canola oil added to each preparation. This is an encouraging outcome that allows further consideration of the phase volumes of the two hydrocolloids in the aqueous domain.

The adaptation of blending laws to study the phase behavior of cold-setting agarose and gelatin in a tertiary system, including soybean oil or Dalda Vanaspati lipid, was described in the literature by Shrinivas et al. [12]. In doing so, theoretical modeling utilised ideas of relating structural morphology to the elastic shear modulus of synthetic polyblends and block polymers. Extensive phase separation in the binary gel of agarose and gelatin was quantified by an estimate of the distribution of water molecules between the two aqueous phases. It is evident in Table 1 that the rapid microscopic protocol of this investigation can be used to probe phase behavior in the tertiary material by arguing that the polysaccharide network occupies about $75 \%$ of the aqueous phase at $3 \%$ (w/w) addition in the mixture with gelatin. In the case of Sample 2, Imaris image analysis returns a result of about one third of the aqueous phase volume being occupied by the WPI network at $5 \%(\mathrm{w} / \mathrm{w})$ addition in mixture with the protein.

\section{Conclusions}

This communication examined the suitability of CLSM based 3D imaging, paired with Imaris image analysis, in estimating the phase behaviour of tertiary systems comprising distinct hydrocolloids and a lipid phase. The combined protocol of microscopy/image analysis was able to provide phase volumes of cold-setting agarose and gelatin in a binary gel with canola oil as the lipid phase. Similarly, reliable predictions were obtained for the phase volumes of thermally treated whey protein isolate in mixture with gelatin and canola oil. The rapid microscopic approach may serve as an alternative to the rather demanding

Table 1 Experimental constituent concentrations in the composites and estimated phase volumes with Imaris image analysis

\begin{tabular}{|c|c|c|}
\hline Materials & $\begin{array}{l}\text { Concentrations added in the } \\
\text { composites }(\%, \mathrm{w} / \mathrm{w})\end{array}$ & $\begin{array}{l}\text { Phase volumes esti- } \\
\text { mated using Imaris } \\
(\%)\end{array}$ \\
\hline \multicolumn{3}{|l|}{ Sample 1} \\
\hline Agarose & 3 & $74.5 \pm 2.1$ \\
\hline Canola oil & 2 & $1.9 \pm 0.3$ \\
\hline Gelatin & 2 & $23.6 \pm 1.7$ \\
\hline \multicolumn{3}{|l|}{ Sample 2} \\
\hline Gelatin & 10 & $60.6 \pm 1.9$ \\
\hline Canola oil & 5 & $5.1 \pm 0.6$ \\
\hline WPI & 5 & $34.3 \pm 1.2$ \\
\hline
\end{tabular}


protocol of rheological experimentation with theoretical blending law analysis that is heavily relied upon to provide predictions of phase volume in phase separated biopolymer networks. Given the increasing need to design complex materials with advanced techno-functionality, the microscopic approach advocated presently might deliver a rapid and accurate methodology to probe and quantify phase morphology in tertiary (and even quaternary) gelling bio-composites.

Acknowledgements The authors wish to acknowledge RMIT Food Research and Innovation Centre for financial support.

Funding Open Access funding enabled and organized by CAUL and its Member Institutions.

\section{Declarations}

Conflicts of Interest/Competing Interests The authors declare no conflict of interests.

Open Access This article is licensed under a Creative Commons Attribution 4.0 International License, which permits use, sharing, adaptation, distribution and reproduction in any medium or format, as long as you give appropriate credit to the original author(s) and the source, provide a link to the Creative Commons licence, and indicate if changes were made. The images or other third party material in this article are included in the article's Creative Commons licence, unless indicated otherwise in a credit line to the material. If material is not included in the article's Creative Commons licence and your intended use is not permitted by statutory regulation or exceeds the permitted use, you will need to obtain permission directly from the copyright holder. To view a copy of this licence, visit http://creativecommons.org/licenses/by/4.0/.

\section{References}

1. Sikorski, Z.E., Pokorny, J. \& Damodaran, S. In Physical and chemical interactions of components in food system. ed. by $\mathrm{S}$. Damodaran, K.L. Parkin, O.R. Fennema, Fennema's Food Chemistry ( $4^{\text {th }}$ ed). (CRC Press, Boca Raton, 2008).
2. M. Takayanagi, H. Harima, Y. Iwata, Viscoelastic behavior of polymer blends and its comparison with model experiments. Memoirs-Facul. Eng. Kyushu Univ. 23, 1-13 (1963)

3. L.E. Nielsen, Morphology and the the elastic modulus of block polymers and polyblends. Rheol. Acta 13, 86-92 (1974)

4. M.A.E. Auty, 4 - Confocal microscopy: Principles and applications to food microstructures, in Food Microstructures. ed. by V.J. Morris, K. Groves (Woodhead Publishing, 2013), pp. 96-P98

5. P. Mhaske, L. Condict, M. Dokouhaki, L. Katopo, S. Kasapis, Quantitative analysis of the phase volume of agarose-canola oil gels in comparison to blending law predictions using 3D imaging based on confocal laser scanning microscopy. Food Res. Int. 125, 108529 (2019)

6. P. Mhaske, L. Condict, M. Dokouhaki, A. Farahnaky, S. Kasapis, Phase volume quantification of agarose-ghee gels using 3D confocal laser scanning microscopy and blending law analysis: A comparison. LWT 129, 109567 (2020)

7. P. Mhaske, A. Farahnaky, S. Kasapis, 3D confocal laser scanning microscopy for quantification of the phase behaviour in agaroseMCC co-gels in comparison to the rheological blending-law analysis. Food Biophys. 16(2), 153-160 (2021)

8. S.K. Ameta, A.K. Rai, D. Hiran, R. Ameta, S.C. Ameta, In Use of nanomaterials in food science. ed. by M. Ghorbanpour, P. Bhargava, A. Varma, \& D. Choudhary. Biogenic nano-particles and their use in Agro-ecosystems. (Springer, Singapore, 2020). https:// doi.org/10.1007/978-981-15-2985-6_24

9. M. Grossi, M. Morgunova, S. Cheung, D. Scholz, E. Conroy, M. Terrile, ..., D.F. O'Shea. Lysosome triggered near-infrared fluorescence imaging of cellular trafficking processes in real time. Nat. Commun. 7(1), 1-13 (2016)

10. M.B. Dürrenberger, S. Handschin, B. Conde-Petit, F. Escher, Visualization of food structure by confocal laser scanning microscopy (CLSM). LWT-Food Sci. Technol. 34(1), 11-17 (2001)

11. W.E. Lorensen, H.E. Cline. Marching cubes: A high resolution 3D surface construction algorithm. Paper presented at the ACM siggraph computer graphics (1987).

12. P. Shrinivas, S. Kasapis, T. Tongdang, Morphology and mechanical properties of bicontinuous gels of agarose and gelatin and the effect of added lipid phase. Langmuir 25(15), 8763-8773 (2009)

Publisher's Note Springer Nature remains neutral with regard to jurisdictional claims in published maps and institutional affiliations. 